\title{
Kinematic Applications of Hyper-Dual Numbers
}

\author{
Selahattin Aslan* \\ (Communicated by Murat Tosun)
}

\begin{abstract}
Hyper-dual numbers are a new number system that is an extension of dual numbers. A hyperdual number can be written uniquely as an ordered pair of dual numbers. In this paper, some basic algebraic properties of hyper-dual numbers are given using their ordered pair representaions of dual numbers. Moreover, the geometric interpretation of a unit hyper-dual vector is given in module as a dual line. And a geometric interpretation of a subset of unit hyper-dual sphere (the set of all unit hyper-dual vectors) is given as two intersecting perpendicular lines in 3-dimensional real vector space.
\end{abstract}

Keywords: Dual numbers, E. Study mapping, hyper-dual numbers, hyper-dual angle.

AMS Subject Classification (2020): Primary: 53A25 ; Secondary: 70B10; $70 E 15$.

\section{Introductions}

The algebra of dual numbers $\mathbb{D}$ was first introduced by W. Clifford in 1873 as an extension of real numbers $\mathbb{R}[2]$. The set of all dual vectors constructs the $\mathbb{D}$-module (also denoted by $\mathbb{D}^{3}$ ). Motion of a rigid body can be represented by two vectors in 3-dimensional real vector space $\mathbb{R}^{3}$. E. Study [11] and A. P. Kotelnikov [10] applied dual numbers in mechanism for the first time by using a dual vector instead of two vectors. In the following years, dual numbers are used in the investigation of instantaneous screw axes with the help of dual transformations in $\mathbb{R}^{3}$ and in Minkowski space $\mathbb{E}_{1}^{3}$ [13-14].

Complex numbers have important advantages in derivative calculations. However, these advantages are lost in the calculations of the second derivative [7]. To overcome this problem, J. A. Fike introduced hyper-dual numbers $\tilde{\mathbb{D}}$ that can be used in the calculation of the first and second derivatives maintaining the advantages of the first derivative by complex numbers [6]. In the following years, J. A. Fike and J. J. Alonso developed this number system for derivative calculations [7, 8]. And it is shown that this number system is suitable for complex software, analysis and design airspace systems, and open kinematic chain robot manipulator [7, 4].

A. Cohen and M. Shoham used hyper-dual numbers in the field of kinematics and dynamics to simplify derivative equations of the motion of multi-body systems [3,4]. They interpreted hyper-dual numbers in the sense of E. Study and A. P. Kotelnikov by using derivative calculations [3-5]. Moreover, they showed that a hyper-dual number can be constituted of two dual numbers [3].

In this paper, some basic concepts of hyper-dual numbers are given using their ordered pair representaions of dual numbers. To give the geometric interpretation of hyper-dual numbers, the concept "dual line" is defined in $\mathbb{D}^{3}$. Also; E. Study mapping is defined in $\mathbb{D}^{3}$, and it is shown that to each unit hyper-dual vector corresponds a dual line in $\mathbb{D}^{3}$. The geometric interpretation of a hyper-dual angle is given as an angle between any two dual lines. Moreover; a subset (denoted by $\tilde{\mathbb{S}}_{1}$ ) of unit hyper-dual sphere $\tilde{\mathbb{S}}$ (the set of all unit hyper-dual vectors) is defined, and it is observed that to each element of $\tilde{\mathbb{S}}_{1}$ corresponds any two intersecting perpendicular directed lines in $\mathbb{R}^{3}$. 


\section{Preliminaries}

In this section a brief summary of the concepts dual and hyper-dual numbers will be given to provide a background to understand the main idea and the results of this study.

\subsection{Dual numbers}

The set of all dual numbers is defined by

$$
\mathbb{D}=\left\{A=a+\varepsilon a^{*}: a, a^{*} \in \mathbb{R}\right\},
$$

where $\varepsilon$ is the dual unit and satisfies

$$
\varepsilon \neq 0, \varepsilon^{2}=0 \text { and } r \varepsilon=\varepsilon r \text { for all } r \in \mathbb{R} .
$$

Addition and multiplication of any dual numbers $A=a+\varepsilon a^{*}$ and $B=b+\varepsilon b^{*}$ are defined, respectively, as

$$
\begin{aligned}
A+B & =(a+b)+\varepsilon\left(a^{*}+b^{*}\right), \\
A B & =a b+\varepsilon\left(a b^{*}+a^{*} b\right) .
\end{aligned}
$$

If $a=1$ and $a^{*}=0$, then $A=1+\varepsilon 0=1$ is called a unit dual number.

The multiplicative-inverse of a dual number $A=a+\varepsilon a^{*}$ is

$$
A^{-1}=\frac{1}{a}-\varepsilon \frac{a^{*}}{a^{2}}, \quad a \neq 0
$$

that means a dual number in the form $A=0+\varepsilon a^{*}=\varepsilon a^{*}$ does not have an multiplicative-inverse.

The square root of a dual number $A=a+\varepsilon a^{*}$ is defined only for the case $a>0$ as

$$
\sqrt{A}=\sqrt{a}+\varepsilon \frac{a^{*}}{2 \sqrt{a}} .
$$

Taylor series expansion of a dual function $f\left(x+\varepsilon x^{*}\right)$ about a point $x+\varepsilon x^{*}=a+\varepsilon a^{*} \in \mathbb{D}$ can be given as

$$
f\left(a+\varepsilon a^{*}\right)=f(a)+\varepsilon a^{*} f^{\prime}(a),
$$

where the prime represents differentiation with respect to $x$, i.e.

$$
f^{\prime}(x)=f^{\prime}(x+\varepsilon 0)=\frac{d}{d x} f(x),
$$

see [12].

Dual numbers form the module

$$
\mathbb{D}^{3}=\left\{\hat{A}=\boldsymbol{a}+\varepsilon \boldsymbol{a}^{*}: \boldsymbol{a}, \boldsymbol{a}^{*} \in \mathbb{R}^{3}\right\},
$$

which is a commutative and associative ring. Each element $\hat{A}$ of $\mathbb{D}^{3}$ is called a dual vector.

The scalar product of any dual vectors $\hat{A}=\boldsymbol{a}+\varepsilon \boldsymbol{a}^{*}$ and $\hat{B}=\boldsymbol{b}+\varepsilon \boldsymbol{b}^{*}$ is defined by

$$
\langle\hat{A}, \hat{B}\rangle_{D}=\langle\boldsymbol{a}, \boldsymbol{b}\rangle+\varepsilon\left(\left\langle\boldsymbol{a}, \boldsymbol{b}^{*}\right\rangle+\left\langle\boldsymbol{a}^{*}, \boldsymbol{b}\right\rangle\right),
$$

where " $\langle$,$\rangle " denotes the usual scalar product in \mathbb{R}^{3}$. It is obvious that $\langle\boldsymbol{a}, \boldsymbol{b}\rangle$ and $\left\langle\boldsymbol{a}, \boldsymbol{b}^{*}\right\rangle+\left\langle\boldsymbol{a}^{*}, \boldsymbol{b}\right\rangle$ are real numbers, and thus $\langle\hat{A}, \hat{B}\rangle_{D}$ is a dual number.

The norm of a dual vector $\hat{A}=\boldsymbol{a}+\varepsilon \boldsymbol{a}^{*}$ is defined to be

$$
N_{\hat{A}}=\langle\hat{A}, \hat{A}\rangle_{D}=|\boldsymbol{a}|^{2}+2 \varepsilon\left\langle\boldsymbol{a}, \boldsymbol{a}^{*}\right\rangle \in \mathbb{D},
$$

where "I,|" denotes the usual modulus in $\mathbb{R}^{3}$. And the modulus (i.e., square root of the norm) of the dual vector $\hat{A}=\boldsymbol{a}+\varepsilon \boldsymbol{a}^{*}$ is defined to be

$$
|\hat{A}|_{D}=\sqrt{\langle\hat{A}, \hat{A}\rangle_{D}}=|\boldsymbol{a}|+\varepsilon \frac{\left\langle\boldsymbol{a}, \boldsymbol{a}^{*}\right\rangle}{|\boldsymbol{a}|}, \text { where }|\boldsymbol{a}| \neq 0 .
$$


If $|\hat{A}|_{D}=1$ (i.e., $|\boldsymbol{a}|=1$ and $\left\langle\boldsymbol{a}, \boldsymbol{a}^{*}\right\rangle=0$ ), then $\hat{A}=\boldsymbol{a}+\varepsilon \boldsymbol{a}^{*}$ is called a unit dual vector.

The vector product of any dual vectors $\hat{A}=\boldsymbol{a}+\varepsilon \boldsymbol{a}^{*}$ and $\hat{B}=\boldsymbol{b}+\varepsilon \boldsymbol{b}^{*}$ is defined by

$$
\hat{A} \times{ }_{D} \hat{B}=\boldsymbol{a} \times \boldsymbol{b}+\varepsilon\left(\boldsymbol{a} \times \boldsymbol{b}^{*}+\boldsymbol{a}^{*} \times \boldsymbol{b}\right),
$$

where " $\times$ " denotes the usual vector product in $\mathbb{R}^{3}$. It is obvious that $\boldsymbol{a} \times \boldsymbol{b}$ and $\boldsymbol{a} \times \boldsymbol{b}^{*}+\boldsymbol{a}^{*} \times \boldsymbol{b}$ are real vectors, and thus $\hat{A} \times{ }_{D} \hat{B}$ is a dual vector.

Unit dual sphere $\mathbb{S}$, consisting of all unit dual vectors, is defined as

$$
\mathbb{S}=\left\{\hat{A}=\boldsymbol{a}+\varepsilon \boldsymbol{a}^{*}:|\hat{A}|_{D}=1, \hat{A} \in \mathbb{D}^{3}\right\} .
$$

Theorem 1. (E. Study Mapping) To each point on unit dual sphere $\mathbb{S}$ corresponds a directed line in $\mathbb{R}^{3}$. In other words, there is a one to one correspondence between the points of unit dual sphere $\mathbb{S}$ and the directed lines in $\mathbb{R}^{3}[11]$.

The geometric interpretation of E. Study mapping can be given as: Let $\hat{A}=\boldsymbol{a}+\varepsilon \boldsymbol{a}^{*}$ be the unit dual vector corresponding to the directed line $d$ in $\mathbb{R}^{3}$. The unit real vector $\boldsymbol{a}$ is the direction vector of the line $d$, and the real vector $\boldsymbol{a}^{*}$ determines the position of $d$, see Figure 1 .

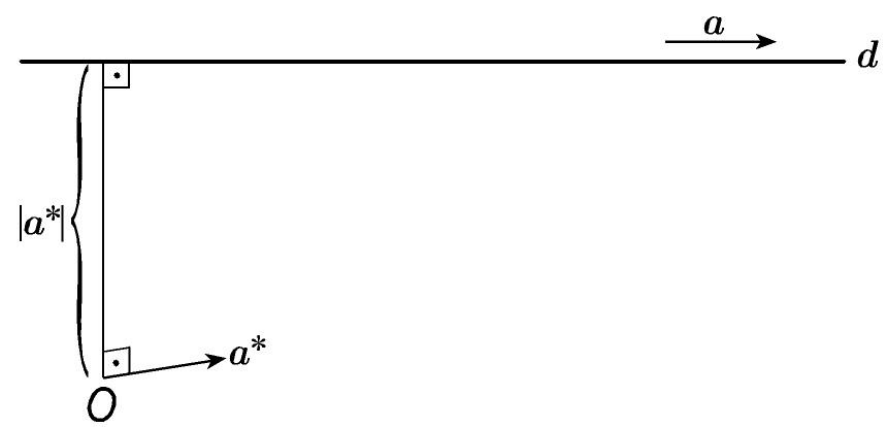

Figure 1. Geometric representation of E. Study mapping in $\mathbb{R}^{3}$

The scalar product of any unit dual vectors $\hat{A}=\boldsymbol{a}+\varepsilon \boldsymbol{a}^{*}$ and $\hat{B}=\boldsymbol{b}+\varepsilon \boldsymbol{b}^{*}$ is obtained as

$$
\langle\hat{A}, \hat{B}\rangle_{D}=\cos \varphi=\cos \theta-\varepsilon \theta^{*} \sin \theta,
$$

where $\varphi=\theta+\varepsilon \theta^{*}$ is a dual angle [11]. If $d_{1}$ and $d_{2}$ are the directed lines in $\mathbb{R}^{3}$ corresponding, respectively, to the unit dual vectors $\hat{A}$ and $\hat{B}$, then $\theta$ is the angle between the real vectors $\boldsymbol{a}$ and $\boldsymbol{b}$, and $\theta^{*}$ is the closest distance between $d_{1}$ and $d_{2}$, see Figure 2 .

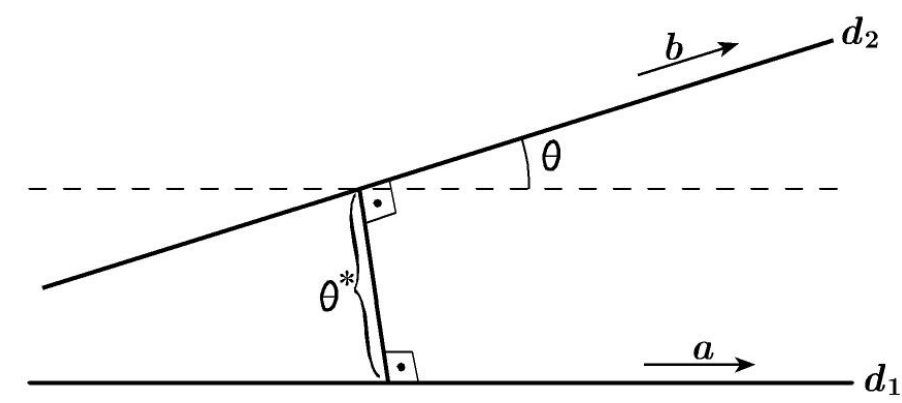

Figure 2. Geometric representation of dual angle between the directed lines $d_{1}$ and $d_{2}$ in $\mathbb{R}^{3}$

The following four cases can be given for a dual angle $\varphi$ satisfying $\cos \varphi=\cos \theta-\varepsilon \theta^{*} \sin \theta$ :

1. If

$$
\cos \theta=0 \text { and } \theta^{*} \neq 0,
$$

then $\theta=\frac{\pi}{2}$ and $\langle\hat{A}, \hat{B}\rangle_{D}=\cos \varphi=-\varepsilon \theta^{*}$. Thus, lines $d_{1}$ and $d_{2}$ are perpendicular but not intersecting. 
2. If

$$
\theta^{*}=0,
$$

then $\langle\hat{A}, \hat{B}\rangle_{D}=\cos \varphi=\cos \theta$. Thus, lines $d_{1}$ and $d_{2}$ are intersecting.

3. If

$$
\langle\hat{A}, \hat{B}\rangle_{D}=\cos \varphi=0
$$

then $\theta=\frac{\pi}{2}$ and $\theta^{*}=0$. Thus, lines $d_{1}$ and $d_{2}$ are perpendicular and intersecting.

4. If

$$
\langle\hat{A}, \hat{B}\rangle_{D}=\cos \varphi=1,
$$

then $\theta=0$. Thus, lines $d_{1}$ and $d_{2}$ are parallel.

The modulus of the vector product of any unit dual vectors $\hat{A}$ and $\hat{B}$ is obtained as

$$
\left|\hat{A} \times_{D} \hat{B}\right|_{D}=\sin \varphi=\sin \theta+\varepsilon \theta^{*} \cos \theta .
$$

For further information about dual numbers, see $[2,12,1]$.

\subsection{Hyper-dual numbers}

The set of all hyper-dual numbers is defined by

$$
\tilde{\mathbb{D}}=\left\{\mathbb{A}=a_{0}+\varepsilon_{1} a_{1}+\varepsilon_{2} a_{2}+\varepsilon_{1} \varepsilon_{2} a_{3}: a_{0}, a_{1}, a_{2}, a_{3} \in \mathbb{R}\right\},
$$

where the dual units $\varepsilon_{1}$ and $\varepsilon_{2}$ satisfy

$$
\varepsilon_{1}^{2}=\varepsilon_{2}^{2}=\left(\varepsilon_{1} \varepsilon_{2}\right)^{2}=0 \text { and } \varepsilon_{1}, \varepsilon_{2}, \varepsilon_{1} \varepsilon_{2} \neq 0
$$

Addition and multiplication of any hyper-dual numbers $\mathbb{A}=a_{0}+\varepsilon_{1} a_{1}+\varepsilon_{2} a_{2}+\varepsilon_{1} \varepsilon_{2} a_{3}$ and $\mathbb{B}=b_{0}+\varepsilon_{1} b_{1}+$ $\varepsilon_{2} b_{2}+\varepsilon_{1} \varepsilon_{2} b_{3}$ are defined, respectively, as

$$
\begin{gathered}
\mathbb{A}+\mathbb{B}=\left(a_{0}+b_{0}\right)+\varepsilon_{1}\left(a_{1}+b_{1}\right)+\varepsilon_{2}\left(a_{2}+b_{2}\right)+\varepsilon_{1} \varepsilon_{2}\left(a_{3}+b_{3}\right), \\
\mathbb{A} \mathbb{B}=\left(a_{0} b_{0}\right)+\varepsilon_{1}\left(a_{0} b_{1}+a_{1} b_{0}\right)+\varepsilon_{2}\left(a_{0} b_{2}+a_{2} b_{0}\right) \\
+\varepsilon_{1} \varepsilon_{2}\left(a_{0} b_{3}+a_{1} b_{2}+a_{2} b_{1}+a_{3} b_{0}\right)
\end{gathered}
$$

The multiplicative-inverse of a hyper-dual number $\mathbb{A}=a_{0}+\varepsilon_{1} a_{1}+\varepsilon_{2} a_{2}+\varepsilon_{1} \varepsilon_{2} a_{3}$ is

$$
\mathbb{A}^{-1}=\frac{1}{\mathbb{A}}=\frac{1}{a_{0}}-\varepsilon_{1} \frac{a_{1}}{a_{0}^{2}}-\varepsilon_{2} \frac{a_{2}}{a_{0}^{2}}+\varepsilon_{1} \varepsilon_{2}\left(-\frac{a_{3}}{a_{0}^{2}}+\frac{2 a_{1} a_{2}}{a_{0}^{3}}\right), a_{0} \neq 0
$$

that means a hyper-dual number in the form $\mathbb{A}=0+\varepsilon_{1} a_{1}+\varepsilon_{2} a_{2}+\varepsilon_{1} \varepsilon_{2} a_{3}=\varepsilon_{1} a_{1}+\varepsilon_{2} a_{2}+\varepsilon_{1} \varepsilon_{2} a_{3}$ does not have an multiplicative-inverse.

Taylor series expansion of a hyper-dual function $f\left(x_{0}+\varepsilon_{1} x_{1}+\varepsilon_{2} x_{2}+\varepsilon_{1} \varepsilon_{2} x_{3}\right)$ about a point $x_{0}+\varepsilon_{1} x_{1}+$ $\varepsilon_{2} x_{2}+\varepsilon_{1} \varepsilon_{2} x_{3}=a_{0}+\varepsilon_{1} a_{1}+\varepsilon_{2} a_{2}+\varepsilon_{1} \varepsilon_{2} a_{3} \in \tilde{\mathbb{D}}$ can be given as

$$
\begin{aligned}
f\left(a_{0}+\varepsilon_{1} a_{1}+\varepsilon_{2} a_{2}+\varepsilon_{1} \varepsilon_{2} a_{3}\right) & =f\left(a_{0}\right)+\varepsilon_{1} a_{1} f^{\prime}\left(a_{0}\right)+\varepsilon_{2} a_{2} f^{\prime}\left(a_{0}\right) \\
& +\varepsilon_{1} \varepsilon_{2}\left(a_{3} f^{\prime}\left(a_{0}\right)+a_{1} a_{2} f^{\prime \prime}\left(a_{0}\right)\right),
\end{aligned}
$$

where the prime represents differentiation with respect to $x_{0}$, i.e.

$$
f^{\prime}\left(x_{0}\right)=f^{\prime}\left(x_{0}+\varepsilon_{1} 0+\varepsilon_{2} 0+\varepsilon_{1} \varepsilon_{2} 0\right)=\frac{d}{d x_{0}} f\left(x_{0}\right),
$$

see [6-9]. 
A hyper-dual number $\mathbb{A}=a_{0}+\varepsilon_{1} a_{1}+\varepsilon_{2} a_{2}+\varepsilon_{1} \varepsilon_{2} a_{3}$ can be given in terms of two dual numbers as

$$
\begin{aligned}
\mathbb{A} & =a_{0}+\varepsilon_{1} a_{1}+\varepsilon_{2} a_{2}+\varepsilon_{1} \varepsilon_{2} a_{3} \\
& =\left(a_{0}+\varepsilon_{1} a_{1}\right)+\varepsilon_{2}\left(a_{2}+\varepsilon_{1} a_{3}\right) \\
& =\left(a_{0}+\varepsilon a_{1}\right)+\varepsilon^{*}\left(a_{2}+\varepsilon a_{3}\right) \\
& =A+\varepsilon^{*} A^{*},
\end{aligned}
$$

where $\varepsilon_{1}=\varepsilon, \varepsilon_{2}=\varepsilon^{*}$ and $A=a_{0}+\varepsilon a_{1}, A^{*}=a_{2}+\varepsilon a_{3} \in \mathbb{D}$.

If we extend the real vectors $\boldsymbol{a}$ and $\boldsymbol{p} \times \boldsymbol{a}$ in a dual vector $\hat{A}=\boldsymbol{a}+\varepsilon(\boldsymbol{p} \times \boldsymbol{a})$, respectively, to the dual vectors $\hat{A}$ and $\hat{P} \times_{D} \hat{A}$, then we obtain the hyper-dual vector

$$
\widetilde{\mathbb{A}}=\hat{A}+\varepsilon^{*}\left(\hat{P} \times_{D} \hat{A}\right) .
$$

Scalar and vector products of any hyper-dual vectors $\widetilde{\mathbb{A}}=\hat{A}+\varepsilon^{*}\left(\hat{P} \times{ }_{D} \hat{A}\right)$ and $\widetilde{\mathbb{B}}=\hat{B}+\varepsilon^{*}\left(\hat{K} \times{ }_{D} \hat{B}\right)$ can be given, respectively, as

$$
\begin{aligned}
\langle\widetilde{\mathbb{A}}, \widetilde{\mathbb{B}}\rangle_{H D} & =|\hat{A}|_{D}|\hat{B}|_{D} \cos \tilde{\varphi} \\
\widetilde{\mathbb{A}} \times_{H D} \widetilde{\mathbb{B}} & =|\hat{A}|_{D}|\hat{B}|_{D} n \sin \tilde{\varphi},
\end{aligned}
$$

where $\tilde{\varphi}$ is a hyper-dual angle and $n$ is the direction vector of the common perpendicular between these two hyper-dual vectors. For further information about hyper-dual numbers, see [3-5].

\section{Applications of Hyper-Dual Numbers in $\mathbb{R}^{3}$ and $\mathbb{D}^{3}$}

In this section, we show that the basic and kinematic concepts of hyper-dual numbers can be given by using dual numbers. Using these concepts, E. Study mapping and hyper-dual angle are abtained in module $\mathbb{D}^{3}$. Furthermore, we have defined a subset (denoted by $\widetilde{\mathbb{S}}_{1}$ ) of unit hyper-dual sphere $\tilde{\mathbb{S}}$ such that to each element of this subset corresponds two intersecting and perpendicular directed lines in $\mathbb{R}^{3}$.

From the definition of a hyper-dual number given by the Equation (2.28), alternative representations of addition (given by Equation (2.23)) and multiplication (given by Equation (2.24)) of any hyper-dual numbers $\mathbb{A}=a_{0}+\varepsilon_{1} a_{1}+\varepsilon_{2} a_{2}+\varepsilon_{1} \varepsilon_{2} a_{3}=A+\varepsilon^{*} A^{*}$ and $\mathbb{B}=b_{0}+\varepsilon_{1} b_{1}+\varepsilon_{2} b_{2}+\varepsilon_{1} \varepsilon_{2} b_{3}=B+\varepsilon^{*} B^{*}$ can be given, respectively, as

$$
\begin{aligned}
\mathbb{A}+\mathbb{B} & =(A+B)+\varepsilon^{*}\left(A^{*}+B^{*}\right), \\
\mathbb{A} \mathbb{B} & =A B+\varepsilon^{*}\left(A B^{*}+A^{*} B\right) .
\end{aligned}
$$

Moreover, an alternative representation of the multiplicative-inverse (given by Equation (2.25)) of a hyper-dual number $\mathbb{A}=a_{0}+\varepsilon_{1} a_{1}+\varepsilon_{2} a_{2}+\varepsilon_{1} \varepsilon_{2} a_{3}=A+\varepsilon^{*} A^{*}$ can be given as

$$
\mathbb{A}^{-1}=\frac{1}{A}-\varepsilon^{*} \frac{A^{*}}{A^{2}}, a_{0} \neq 0
$$

that means a hyper-dual number $\mathbb{A}=A+\varepsilon^{*} A^{*}$ providing $A=0+\varepsilon a_{1}=\varepsilon a_{1}$ does not have an multiplicativeinverse.

The square root of a hyper-dual number $\mathbb{A}=A+\varepsilon^{*} A^{*}$ can be defined by

$$
\sqrt{\mathbb{A}}=\sqrt{A}+\varepsilon^{*} \frac{A^{*}}{2 \sqrt{A}}, a_{0}>0
$$

or

$$
\sqrt{\mathbb{A}}=\sqrt{a_{0}}+\varepsilon_{1} \frac{a_{1}}{2 \sqrt{a_{0}}}+\varepsilon_{2} \frac{a_{2}}{2 \sqrt{a_{0}}}+\varepsilon_{1} \varepsilon_{2}\left(\frac{a_{3}}{2 \sqrt{a_{0}}}-\frac{a_{1} a_{2}}{4 a_{0} \sqrt{a_{0}}}\right), a_{0}>0 .
$$


An alternative representation of the Taylor series expension of a hyper-dual function given by Equation (2.26) can be given by the following theorem.

Theorem 2. Let $\mathbb{A}=A+\varepsilon^{*} A^{*}$ be a hyper-dual number, where $A=a_{0}+\varepsilon a_{1}, A^{*}=a_{2}+\varepsilon a_{3} \in \mathbb{D}$. Then, the Taylor series expansion of the hyper-dual function $f\left(x_{0}+\varepsilon x_{1}+\varepsilon^{*} x_{2}+\varepsilon \varepsilon^{*} x_{3}\right)$ about a point $x_{0}+\varepsilon x_{1}+\varepsilon^{*} x_{2}+\varepsilon \varepsilon^{*} x_{3}=$ $a_{0}+\varepsilon a_{1}+\varepsilon^{*} a_{2}+\varepsilon \varepsilon^{*} a_{3} \in \tilde{\mathbb{D}}$ can be given as

$$
f\left(A+\varepsilon^{*} A^{*}\right)=f(A)+\varepsilon^{*} A^{*} f^{\prime}(A),
$$

where $f^{\prime}(A)=f^{\prime}\left(a_{0}+\varepsilon a_{1}\right)$ is the first derivative of the dual function $f\left(x_{0}+\varepsilon x_{1}\right)$ with respect to $x_{0}$ at the point $x_{0}+\varepsilon x_{1}=a_{0}+\varepsilon a_{1} \in \mathbb{D}$, i.e.

$$
f^{\prime}\left(x_{0}\right)=f^{\prime}\left(x_{0}+\varepsilon 0\right)=\frac{d}{d x_{0}} f\left(x_{0}\right) .
$$

Proof. From Equation (2.7), the Taylor series expansions of $f(A)$ and $f^{\prime}(A)$ can be given, respectively, as

$$
\begin{aligned}
f(A) & =f\left(a_{0}+\varepsilon a_{1}\right)=f\left(a_{0}\right)+\varepsilon a_{1} f^{\prime}\left(a_{0}\right), \\
f^{\prime}(A) & =f^{\prime}\left(a_{0}+\varepsilon a_{1}\right)=f^{\prime}\left(a_{0}\right)+\varepsilon a_{1} f^{\prime \prime}\left(a_{0}\right),
\end{aligned}
$$

where the prime represents differentiation with respect to $x_{0}$, i.e.

$$
\begin{aligned}
& f^{\prime}\left(x_{0}\right)=f^{\prime}\left(x_{0}+\varepsilon 0\right)=\frac{d}{d x_{0}} f\left(x_{0}\right), \\
& f^{\prime \prime}\left(x_{0}\right)=f^{\prime \prime}\left(x_{0}+\varepsilon 0\right)=\frac{d}{d x_{0}} f^{\prime}\left(x_{0}\right) .
\end{aligned}
$$

Using the Equation (2.26), we get

$$
\begin{aligned}
f(\mathbb{A}) & =f\left(a_{0}\right)+\varepsilon a_{1} f^{\prime}\left(a_{0}\right)+\varepsilon^{*} a_{2} f^{\prime}\left(a_{0}\right)+\varepsilon \varepsilon^{*}\left(a_{3} f^{\prime}\left(a_{0}\right)+a_{1} a_{2} f^{\prime \prime}\left(a_{0}\right)\right) \\
& =\left(f\left(a_{0}\right)+\varepsilon a_{1} f^{\prime}\left(a_{0}\right)\right)+\varepsilon^{*}\left(a_{2} f^{\prime}\left(a_{0}\right)+\varepsilon\left(a_{3} f^{\prime}\left(a_{0}\right)+a_{1} a_{2} f^{\prime \prime}\left(a_{0}\right)\right)\right) \\
& =\left(f\left(a_{0}\right)+\varepsilon a_{1} f^{\prime}\left(a_{0}\right)\right)+\varepsilon^{*}\left(a_{2} f^{\prime}\left(a_{0}\right)+\varepsilon a_{1} a_{2} f^{\prime \prime}\left(a_{0}\right)+\varepsilon a_{3} f^{\prime}\left(a_{0}\right)\right) \\
& =\left(f\left(a_{0}\right)+\varepsilon a_{1} f^{\prime}\left(a_{0}\right)\right)+\varepsilon^{*}\left(a_{2} f^{\prime}\left(a_{0}\right)+\varepsilon a_{1} a_{2} f^{\prime \prime}\left(a_{0}\right)+\varepsilon a_{3} f^{\prime}\left(a_{0}\right)\right. \\
& \left.+\varepsilon^{2} a_{1} a_{3} f^{\prime \prime}\left(a_{0}\right)\right) \\
& =\left(f\left(a_{0}\right)+\varepsilon a_{1} f^{\prime}\left(a_{0}\right)\right)+\varepsilon^{*}\left(a_{2}\left(f^{\prime}\left(a_{0}\right)+\varepsilon a_{1} f^{\prime \prime}\left(a_{0}\right)\right)+\varepsilon a_{3}\left(f^{\prime}\left(a_{0}\right)\right.\right. \\
& \left.\left.+\varepsilon a_{1} f^{\prime \prime}\left(a_{0}\right)\right)\right) \\
& =\left(f\left(a_{0}\right)+\varepsilon a_{1} f^{\prime}\left(a_{0}\right)\right)+\varepsilon^{*}\left(a_{2}+\varepsilon a_{3}\right)\left(f^{\prime}\left(a_{0}\right)+\varepsilon a_{1} f^{\prime \prime}\left(a_{0}\right)\right) .
\end{aligned}
$$

Inserting Equations (3.8) and (3.9) in the Equation (3.12), we also get

$$
f\left(A+\varepsilon^{*} A^{*}\right)=f(A)+\varepsilon^{*}\left(a_{2}+\varepsilon a_{3}\right) f^{\prime}(A),
$$

and using $A^{*}=a_{2}+\varepsilon a_{3}$, we obtain

$$
f\left(A+\varepsilon^{*} A^{*}\right)=f(A)+\varepsilon^{*} A^{*} f^{\prime}(A) .
$$

We need to define the concept line in $\mathbb{D}^{3}$ to give the geometric interpretations of hyper-dual numbers in $\mathbb{D}^{3}$.

Definition 1. (Dual line) Let $\hat{A}$ be a unit dual vector and $\hat{P}$ be a point in $\mathbb{D}^{3}$. Then, a line in $\mathbb{D}^{3}$ can be defined by

$$
\hat{d}=\hat{P}+T \hat{A},
$$

where the parameter $T$ is a dual number, the unit dual vector $\hat{A}$ is the direction vector of $\hat{d}$, and $\hat{P}$ is a point on $\hat{d}$. We will call a line in $\mathbb{D}^{3}$ as dual line. 
Definition 2. (Hyper-dual vectors) The set of all hyper-dual vectors is defined by

$$
\begin{aligned}
\tilde{\mathbb{D}}^{3} & =\left\{\widetilde{\mathbb{A}}=\hat{A}+\varepsilon^{*} \hat{A}^{*}: \hat{A}, \hat{A}^{*} \in \mathbb{D}^{3}\right\} \\
& =\left\{\widetilde{\mathbb{A}}=\boldsymbol{a}_{0}+\varepsilon \boldsymbol{a}_{1}+\varepsilon^{*} \boldsymbol{a}_{2}+\varepsilon \varepsilon^{*} \boldsymbol{a}_{3}: \boldsymbol{a}_{0}, \boldsymbol{a}_{1}, \boldsymbol{a}_{2}, \boldsymbol{a}_{3} \in \mathbb{R}^{3}\right\},
\end{aligned}
$$

and each element $\widetilde{\mathbb{A}}$ of $\tilde{\mathbb{D}}^{3}$ is called a hyper-dual vector.

The scalar and vector products of any hyper-dual vectors $\widetilde{\mathbb{A}}=\hat{A}+\varepsilon^{*} \hat{A}^{*}=\boldsymbol{a}_{0}+\varepsilon \boldsymbol{a}_{1}+\varepsilon^{*} \boldsymbol{a}_{2}+\varepsilon \varepsilon^{*} \boldsymbol{a}_{3}$ and $\widetilde{\mathbb{B}}=\hat{B}+\varepsilon^{*} \hat{B}^{*}=\boldsymbol{b}_{0}+\varepsilon \boldsymbol{b}_{1}+\varepsilon^{*} \boldsymbol{b}_{2}+\varepsilon \varepsilon^{*} \boldsymbol{b}_{3}$ are defined, respectively, by

$$
\begin{aligned}
\langle\widetilde{\mathbb{A}}, \widetilde{\mathbb{B}}\rangle_{H D} & =\langle\hat{A}, \hat{B}\rangle_{D}+\varepsilon^{*}\left(\left\langle\hat{A}, \hat{B}^{*}\right\rangle_{D}+\left\langle\hat{A}^{*}, \hat{B}\right\rangle_{D}\right) \\
& =\left\langle\boldsymbol{a}_{0}, \boldsymbol{b}_{0}\right\rangle+\varepsilon\left(\left\langle\boldsymbol{a}_{0}, \boldsymbol{b}_{1}\right\rangle+\left\langle\boldsymbol{a}_{1}, \boldsymbol{b}_{0}\right\rangle\right)+\varepsilon^{*}\left(\left\langle\boldsymbol{a}_{0}, \boldsymbol{b}_{2}\right\rangle+\left\langle\boldsymbol{a}_{2}, \boldsymbol{b}_{0}\right\rangle\right) \\
& +\varepsilon \varepsilon^{*}\left(\left\langle\boldsymbol{a}_{0}, \boldsymbol{b}_{3}\right\rangle+\left\langle\boldsymbol{a}_{1}, \boldsymbol{b}_{2}\right\rangle+\left\langle\boldsymbol{a}_{2}, \boldsymbol{b}_{1}\right\rangle+\left\langle\boldsymbol{a}_{3}, \boldsymbol{b}_{0}\right\rangle\right), \\
\widetilde{\mathbb{A}} \times_{H D} \widetilde{\mathbb{B}} & =\hat{A} \times_{D} \hat{B}+\varepsilon^{*}\left(\hat{A} \times_{D} \hat{B}^{*}+\hat{A}^{*} \times_{D} \hat{B}\right) . \\
& =\boldsymbol{a}_{0} \times \boldsymbol{b}_{0}+\varepsilon\left(\boldsymbol{a}_{0} \times \boldsymbol{b}_{1}+\boldsymbol{a}_{1} \times \boldsymbol{b}_{0}\right)+\varepsilon^{*}\left(\boldsymbol{a}_{0} \times \boldsymbol{b}_{2}+\boldsymbol{a}_{2} \times \boldsymbol{b}_{0}\right) \\
& +\varepsilon \varepsilon^{*}\left(\boldsymbol{a}_{0} \times \boldsymbol{b}_{3}+\boldsymbol{a}_{1} \times \boldsymbol{b}_{2}+\boldsymbol{a}_{2} \times \boldsymbol{b}_{1}+\boldsymbol{a}_{3} \times \boldsymbol{b}_{0}\right) .
\end{aligned}
$$

Since $\langle\hat{A}, \hat{B}\rangle_{D}$ and $\left\langle\hat{A}, \hat{B}^{*}\right\rangle_{D}+\left\langle\hat{A}^{*}, \hat{B}\right\rangle_{D}$ are dual numbers, $\langle\widetilde{\mathbb{A}}, \widetilde{\mathbb{B}}\rangle_{H D}$ is a hyper-dual number. And since $\hat{A} \times{ }_{D} \hat{B}$ and $\hat{A} \times_{D} \hat{B}^{*}+\hat{A}^{*} \times_{D} \hat{B}$ are dual vectors, $\widetilde{\mathbb{A}} \times_{H D} \widetilde{\mathbb{B}}$ is a hyper-dual vector.

The norm of a hyper-dual vector $\widetilde{\mathbb{A}}=\hat{A}+\varepsilon^{*} \hat{A}^{*}=\boldsymbol{a}_{0}+\varepsilon \boldsymbol{a}_{1}+\varepsilon^{*} \boldsymbol{a}_{2}+\varepsilon \varepsilon^{*} \boldsymbol{a}_{3}$ is defined to be

$$
\begin{aligned}
N_{\widetilde{\mathbb{A}}} & =\langle\widetilde{\mathbb{A}}, \widetilde{\mathbb{A}}\rangle_{H D}=|\hat{A}|_{D}^{2}+2 \varepsilon^{*}\left\langle\hat{A}, \hat{A}^{*}\right\rangle_{D} \\
& =\left|\boldsymbol{a}_{0}\right|^{2}+2\left(\varepsilon\left\langle\boldsymbol{a}_{0}, \boldsymbol{a}_{1}\right\rangle+\varepsilon^{*}\left\langle\boldsymbol{a}_{0}, \boldsymbol{a}_{2}\right\rangle+\varepsilon \varepsilon^{*}\left(\left\langle\boldsymbol{a}_{0}, \boldsymbol{a}_{3}\right\rangle+\left\langle\boldsymbol{a}_{1}, \boldsymbol{a}_{2}\right\rangle\right)\right) .
\end{aligned}
$$

And the modulus (i.e., square root of the norm) of the hyper-dual vector $\widetilde{\mathbb{A}}$ is defined to be

$$
\begin{aligned}
|\widetilde{\mathbb{A}}|_{H D} & =\sqrt{\langle\widetilde{\mathbb{A}}, \widetilde{\mathbb{A}}\rangle_{H D}}=|\hat{A}|_{D}+\varepsilon^{*} \frac{\left\langle\hat{A}, \hat{A}^{*}\right\rangle_{D}}{|\hat{A}|_{D}} \\
& =\left|\boldsymbol{a}_{0}\right|+\varepsilon \frac{\left\langle\boldsymbol{a}_{0}, \boldsymbol{a}_{1}\right\rangle}{\left|\boldsymbol{a}_{0}\right|}+\varepsilon^{*} \frac{\left\langle\boldsymbol{a}_{0}, \boldsymbol{a}_{2}\right\rangle}{\left|\boldsymbol{a}_{0}\right|} \\
& +\varepsilon \varepsilon^{*}\left(\frac{\left\langle\boldsymbol{a}_{0}, \boldsymbol{a}_{3}\right\rangle}{\left|\boldsymbol{a}_{0}\right|}+\frac{\left\langle\boldsymbol{a}_{1}, \boldsymbol{a}_{2}\right\rangle}{\left|\boldsymbol{a}_{0}\right|}-\frac{\left\langle\boldsymbol{a}_{0}, \boldsymbol{a}_{1}\right\rangle\left\langle\boldsymbol{a}_{0}, \boldsymbol{a}_{2}\right\rangle}{\left|\boldsymbol{a}_{0}\right|^{3}}\right),
\end{aligned}
$$

where $\left|\boldsymbol{a}_{0}\right| \neq 0$.

If $|\widetilde{\mathbb{A}}|_{H D}=1$ (i.e., $|\hat{A}|_{D}=1$ and $\left\langle\hat{A}, \hat{A}^{*}\right\rangle_{D}=0$ ), then $\widetilde{\mathbb{A}}=\hat{A}+\varepsilon^{*} \hat{A}^{*}$ is called a unit hyper-dual vector.

Definition 3. (Unit hyper-dual sphere) Unit hyper-dual sphere $\tilde{\mathbb{S}}$, consisting of all unit hyper-dual vectors, can be defined as

$$
\tilde{\mathbb{S}}=\left\{\widetilde{\mathbb{A}}=\hat{A}+\varepsilon^{*} \hat{A}^{*}:|\widetilde{\mathbb{A}}|_{H D}=1 ; \hat{A}, \hat{A}^{*} \in \mathbb{D}^{3}\right\} .
$$

Theorem 3. (E. Study mapping for unit hyper-dual vectors) To each point on unit hyper-dual sphere $\tilde{\mathbb{S}}$ corresponds a directed dual line $\hat{d}$ in $\mathbb{D}^{3}$. In other words, there is a one to one correspondence between the points of unit hyper-dual sphere $\tilde{\mathbb{S}}$ and the directed dual lines in $\mathbb{D}^{3}$.

Proof. A directed line in $\mathbb{D}^{3}$ (i.e., directed dual line) can be given by any two points $\hat{X}$ and $\hat{Y}$ on it. The parametric equation of this dual line is

$$
\hat{Y}=\hat{X}+T \hat{A},
$$

where $T$ is a non-zero dual constant and $\hat{A}$ is a unit dual vector. The moment of the vector $\hat{A}$ with respect to the origin $\hat{O}$ is

$$
\hat{A}^{*}=\hat{X} \times_{D} \hat{A}=\hat{Y} \times_{D} \hat{A}
$$


That means; the direction vector $\hat{A}$ of the dual line and its moment vector $\hat{A}^{*}$ are independent of choice of the points of the dual line. The two vectors $\hat{A}$ and $\hat{A}^{*}$ are not independent of one another; so they satisfy the equations

$$
|\hat{A}|_{D}=1 \text { and }\left\langle\hat{A}, \hat{A}^{*}\right\rangle_{D}=0 .
$$

The six dual components $A_{i}, A_{i}^{*}$ (for $i=1,2,3$ ) of $\hat{A}$ and $\hat{A}^{*}$ are Plückerian homogeneous dual line coordinates. Hence the two dual vectors $\hat{A}$ and $\hat{A}^{*}$ determine the directed dual line. A point $\hat{Z}$ is on the dual line of dual vectors $\hat{A}$ and $\hat{A}^{*}$ if and only if

$$
\hat{Z} \times{ }_{D} \hat{A}=\hat{A}^{*}
$$

The set of directed dual lines is in one to one correspondence with pairs of dual vectors in $\mathbb{D}^{3}$ subject to the conditions (given by Equation (3.27)). Consequently; since $\hat{A}$ is a unit dual vector (i.e., $|\hat{A}|_{D}=1$ ) and $\left\langle\hat{A}, \hat{A}^{*}\right\rangle_{D}=0$, the unit hyper-dual vector $\widetilde{\mathbb{A}}=\hat{A}+\varepsilon^{*} \hat{A}^{*}$ represents a dual line, see Figure 3.

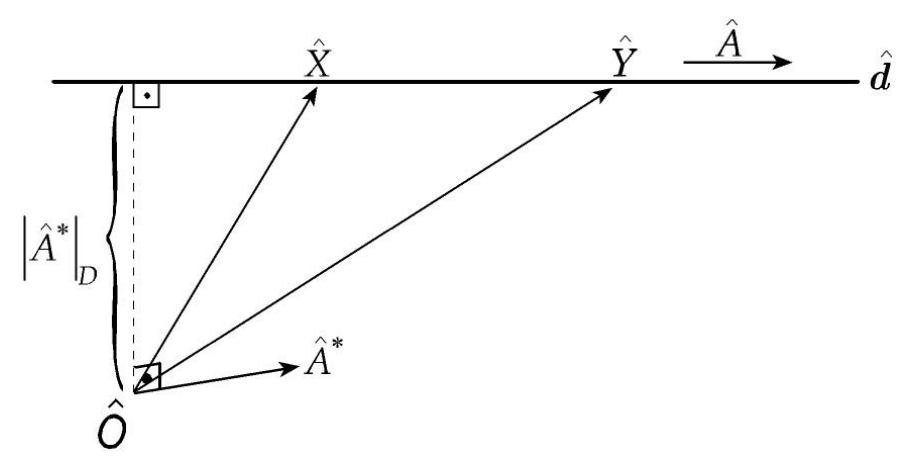

Figure 3. Geometric representation of E. Study mapping in $\mathbb{D}^{3}$

Example 1. (Application of E. Study mapping for unit hyper-dual vectors) Let us take the unit hyperdual vector $\widetilde{\mathbb{A}}=\left(\frac{1}{\sqrt{3}}, \frac{1}{\sqrt{3}}+\varepsilon, \frac{1}{\sqrt{3}}-\varepsilon\right)+\varepsilon^{*}(-2+\varepsilon, 1,1-\varepsilon)$ that can be written in the form $\widetilde{\mathbb{A}}=\hat{A}+\varepsilon^{*} \hat{A}^{*}$ for $\hat{A}=\left(\frac{1}{\sqrt{3}}, \frac{1}{\sqrt{3}}+\varepsilon, \frac{1}{\sqrt{3}}-\varepsilon\right)$ and $\hat{A}^{*}=(-2+\varepsilon, 1,1-\varepsilon)$. If $\hat{d}$ is the corresponding dual line in $\mathbb{D}^{3}$ to $\widetilde{\mathbb{A}}$, and $\hat{Z}$ is the nearest point from the origin $\hat{O}$ to the line $\hat{d}$, then the equalities

$$
\hat{Z} \times_{D} \hat{A}=\hat{A}^{*} \text { and }\langle\hat{Z}, \hat{A}\rangle_{D}=\left\langle\hat{Z}, \hat{A}^{*}\right\rangle_{D}=0
$$

can be given. From these equations, we get

$$
\hat{Z}=\left(\varepsilon\left(2-\frac{1}{\sqrt{3}}\right),-\sqrt{3}+\varepsilon\left(2+\frac{2}{\sqrt{3}}\right), \sqrt{3}+\varepsilon\left(2-\frac{1}{\sqrt{3}}\right)\right) .
$$

Since the unit dual vector $\hat{A}$ is the direction vector of $\hat{d}$, and $\hat{Z}$ is a point on $\hat{d}$, we can give the corresponding dual line to unit hyper-dual vector $\widetilde{\mathbb{A}}$ as

$$
\begin{aligned}
\hat{d} & =\left(\varepsilon\left(2-\frac{1}{\sqrt{3}}\right),-\sqrt{3}+\varepsilon\left(2+\frac{2}{\sqrt{3}}\right), \sqrt{3}+\varepsilon\left(2-\frac{1}{\sqrt{3}}\right)\right) \\
& +T\left(\frac{1}{\sqrt{3}}, \frac{1}{\sqrt{3}}+\varepsilon, \frac{1}{\sqrt{3}}-\varepsilon\right),
\end{aligned}
$$

where the parameter $T$ is a dual variable.

Theorem 4. Let us take a subset of unit hyper-dual sphere $\tilde{\mathbb{S}}$ as

$$
\tilde{\mathbb{S}}_{1}=\left\{\widetilde{\mathbb{A}}=\hat{A}+\varepsilon^{*} \hat{A}^{*}:\left|\hat{A}^{*}\right|_{D}=1, \widetilde{\mathbb{A}} \in \tilde{\mathbb{S}}\right\}
$$

Then, there exists a one to one correspondence between the points of $\tilde{\mathbb{S}}_{1}$ and any two intersecting perpendicular directed lines in $\mathbb{R}^{3}$. 
Proof. Since $\widetilde{\mathbb{A}} \in \tilde{\mathbb{S}}_{1} ; \hat{A}$ and $\hat{A}^{*}$ are unit dual vectors and $\widetilde{\mathbb{A}}=\hat{A}+\varepsilon^{*} \hat{A}^{*}$ is a unit hyper-dual vector satisfying $|\hat{A}|_{D}=1$ and $\left\langle\hat{A}, \hat{A}^{*}\right\rangle_{D}=0$. According to Theorem 1 , let $\hat{A}$ and $\hat{A}^{*}$ represent the directed lines $d_{1}$ and $d_{2}$ in $\mathbb{R}^{3}$, respectively. Thus, from Equation (2.18), the property $\left\langle\hat{A}, \hat{A}^{*}\right\rangle_{D}=0$ shows that $d_{1}$ and $d_{2}$ are perpendicular intersecting directed lines.

Example 2. (Application of the subset $\left.\tilde{\mathbb{S}}_{1}\right)$ Let us take the unit hyper-dual vector $\widetilde{\mathbb{A}}=(\varepsilon, 1,0)+\varepsilon^{*}(-\varepsilon, 0,1)$ that can be written in the form $\widetilde{\mathbb{A}}=\hat{A}+\varepsilon^{*} \hat{A}^{*}$ for

$$
\begin{aligned}
\hat{A} & =(\varepsilon, 1,0)=(0,1,0)+\varepsilon(1,0,0), \\
\hat{A}^{*} & =(-\varepsilon, 0,1)=(0,0,1)+\varepsilon(-1,0,0) .
\end{aligned}
$$

Since $|\hat{A}|_{D}=\left|\hat{A}^{*}\right|_{D}=1 ; \hat{A}$ and $\hat{A}^{*}$ are unit dual vectors, and thus $\widetilde{\mathbb{A}} \in \tilde{\mathbb{S}}_{1}$. According to Theorem 4, unit hyperdual vector $\widetilde{\mathbb{A}}$ represents two perpendicular intersecting directed lines in $\mathbb{R}^{3}$. And according to E. Study mapping, each of these lines correspond to a unit dual vector (one of them corresponds to $\hat{A}$ and the other to $\hat{A}^{*}$ ), [11]. These lines will be obtained, respectively, as

$$
\begin{aligned}
& d_{1}=(0,0,-1)+t_{1}(0,1,0), \\
& d_{2}=(0,-1,0)+t_{2}(0,0,1),
\end{aligned}
$$

where the parameters $t_{1}$ and $t_{2}$ are real variables. Direction vectors of $d_{1}$ and $d_{2}$ are $\boldsymbol{v}_{1}=(0,1,0)$ and $\boldsymbol{v}_{2}=$ $(0,0,1)$, respectively. Since $\left\langle\boldsymbol{v}_{1}, \boldsymbol{v}_{2}\right\rangle=0 ; d_{1}$ and $d_{2}$ are perpendicular. And for $t_{1}=-1$ and $t_{2}=-1 ; d_{1}$ and $d_{2}$ intersect at the point $(0,-1,-1)$.

\section{Definition 4. (Hyper-dual angle)}

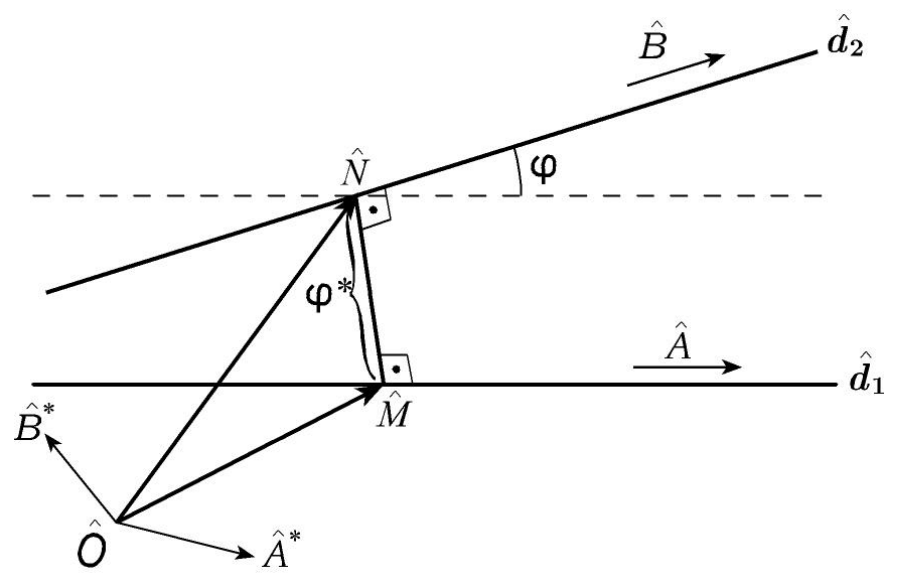

Figure 4. Geometric representation of hyper-dual angle between the directed dual lines $\hat{d}_{1}$ and $\hat{d}_{2}$ in $\mathbb{D}^{3}$

The scalar product of any unit hyper-dual vectors $\widetilde{\mathbb{A}}=\hat{A}+\varepsilon^{*} \hat{A}^{*}$ and $\widetilde{\mathbb{B}}=\hat{B}+\varepsilon^{*} \hat{B}^{*}$ is

$$
\langle\widetilde{\mathbb{A}}, \widetilde{\mathbb{B}}\rangle_{H D}=\langle\hat{A}, \hat{B}\rangle_{D}+\varepsilon^{*}\left(\left\langle\hat{A}, \hat{B}^{*}\right\rangle_{D}+\left\langle\hat{A}^{*}, \hat{B}\right\rangle_{D}\right) .
$$

If $\hat{d}_{1}$ and $\hat{d}_{2}$ are the directed dual lines in $\mathbb{D}^{3}$ corresponding, respectively, to the unit hyper-dual vectors $\widetilde{\mathbb{A}}$ and $\widetilde{\mathbb{B}}$, then then, $\hat{A}$ and $\hat{A}^{*}$ represent, respectively, the direction vector and the position of $\hat{d}_{1}$; and $\hat{B}, \hat{B}^{*}$ represent, respectively, the direction vector and the position of $\hat{d}_{2}$ in $\mathbb{D}^{3}$. In Equation (3.39), $\langle\hat{A}, \hat{B}\rangle_{D}$ is equal to

$$
\langle\hat{A}, \hat{B}\rangle_{D}=\cos \varphi,
$$


where $\varphi$ is the dual angle between the unit dual vectors $\hat{A}$ and $\hat{B}$. Let $\hat{M} \in \hat{d}_{1}$ and $\hat{N} \in \hat{d}_{2}$ be the two closest points on $\hat{d}_{1}$ and $\hat{d}_{2}$. Then, using $\hat{M} \times_{D} \hat{A}=\hat{A}^{*}$ and $\hat{N} \times{ }_{D} \hat{B}=\hat{B}^{*}$ in Equation (3.39), we get

$$
\begin{aligned}
\left\langle\hat{A}, \hat{B}^{*}\right\rangle_{D}+\left\langle\hat{A}^{*}, \hat{B}\right\rangle_{D} & =\left\langle\hat{A},\left(\hat{N} \times_{D} \hat{B}\right)\right\rangle_{D}+\left\langle\left(\hat{M} \times_{D} \hat{A}\right), \hat{B}\right\rangle_{D} \\
& =-\left\langle\hat{N},\left(\hat{A} \times_{D} \hat{B}\right)\right\rangle_{D}+\left\langle\hat{M},\left(\hat{A} \times_{D} \hat{B}\right)\right\rangle_{D} \\
& =\left\langle(\hat{M}-\hat{N}),\left(\hat{A} \times_{D} \hat{B}\right)\right\rangle_{D} .
\end{aligned}
$$

Let $\varphi^{*}$ be the distance between the points $\hat{M}$ and $\hat{N}$, then we can write

$$
|\hat{M}-\hat{N}|_{D}=\varphi^{*}
$$

It is obvious that $\varphi^{*}$ is a dual number, because the modulus of $\hat{M}$ and $\hat{N}$ are dual numbers (see Equation (2.12)). Using $|\hat{M}-\hat{N}|_{D}=\varphi^{*}$ in Equation (3.41), we get

$$
\begin{aligned}
\left\langle(\hat{M}-\hat{N}),\left(\hat{A} \times_{D} \hat{B}\right)\right\rangle_{D} & =\left\langle\left(\frac{\hat{M}-\hat{N}}{|\hat{M}-\hat{N}|_{D}} \varphi^{*}\right),\left(\hat{A} \times_{D} \hat{B}\right)\right\rangle_{D} \\
& =\left\langle\left(\frac{\hat{A} \times{ }_{D} \hat{B}}{\left|\hat{A} \times{ }_{D} \hat{B}\right|_{D}} \varphi^{*}\right),\left(\hat{A} \times{ }_{D} \hat{B}\right)\right\rangle_{D} \\
& =\frac{\varphi^{*}}{\left|\hat{A} \times{ }_{D} \hat{B}\right|_{D}}\left\langle\left(\hat{A} \times{ }_{D} \hat{B}\right),\left(\hat{A} \times{ }_{D} \hat{B}\right)\right\rangle_{D} \\
& = \pm \varphi^{*}\left|\hat{A} \times{ }_{D} \hat{B}\right|_{D} \\
& = \pm \varphi^{*} \sin \varphi .
\end{aligned}
$$

Inserting Equations (3.40) and (3.43) in Equation (3.39), we also get

$$
\begin{aligned}
\langle\widetilde{\mathbb{A}}, \widetilde{\mathbb{B}}\rangle_{H D} & =\langle\hat{A}, \hat{B}\rangle_{D}+\varepsilon^{*}\left(\left\langle\hat{A}, \hat{B}^{*}\right\rangle_{D}+\left\langle\hat{A}^{*}, \hat{B}\right\rangle_{D}\right) \\
& =\cos \varphi-\varepsilon^{*} \varphi^{*} \sin \varphi .
\end{aligned}
$$

By using the Taylor series expansion given by Equation (3.6) in Equation (3.44), we obtain

$$
\langle\widetilde{\mathbb{A}}, \widetilde{\mathbb{B}}\rangle_{H D}=\cos \varphi-\varepsilon^{*} \varphi^{*} \sin \varphi=\cos \tilde{\varphi}
$$

where $\tilde{\varphi}=\varphi+\varepsilon^{*} \varphi^{*}$ is a hyper dual angle, see Figure 4. Similarly, the modulus of the vector product of any unit hyper-dual vectors $\widetilde{\mathbb{A}}=\hat{A}+\varepsilon^{*} \hat{A}^{*}$ and $\widetilde{\mathbb{B}}=\hat{B}+\varepsilon^{*} \hat{B}^{*}$ can be given as

$$
\left|\widetilde{\mathbb{A}} \times_{H D} \widetilde{\mathbb{B}}\right|_{H D}=\sin \varphi+\varepsilon^{*} \varphi^{*} \cos \varphi=\sin \tilde{\varphi} .
$$

Proposition 1. If $\widetilde{\mathbb{A}}$ and $\widetilde{\mathbb{B}}$ are hyper-dual vectors, then

$$
\widetilde{\mathbb{V}}=\frac{\widetilde{\mathbb{A}}}{|\widetilde{\mathbb{A}}|_{H D}} \text { and } \widetilde{\mathbb{U}}=\frac{\widetilde{\mathbb{B}}}{|\widetilde{\mathbb{B}}|_{H D}}
$$

are unit hyper-dual vectors. From the equations

$$
\begin{aligned}
\langle\widetilde{\mathbb{V}}, \widetilde{\mathbb{U}}\rangle_{H D} & =\cos \tilde{\varphi}, \\
\left|\widetilde{\mathbb{V}} \times_{H D} \widetilde{\mathbb{U}}\right|_{H D} & =\sin \tilde{\varphi}
\end{aligned}
$$


we can give

$$
\begin{aligned}
\langle\widetilde{\mathbb{A}}, \widetilde{\mathbb{B}}\rangle_{H D} & =|\widetilde{\mathbb{A}}|_{H D}|\widetilde{\mathbb{B}}|_{H D} \cos \tilde{\varphi}, \\
\left|\widetilde{\mathbb{A}} \times_{H D} \widetilde{\mathbb{B}}\right|_{H D} & =|\widetilde{\mathbb{A}}|_{H D}|\widetilde{\mathbb{B}}|_{H D} \sin \tilde{\varphi},
\end{aligned}
$$

where $\tilde{\varphi}$ is a hyper-dual angle.

Let $\tilde{\varphi}, \varphi$ and $\theta$ be, respectively, a hyper-dual angle, a dual angle and a real angle. Then, the following four cases can be given related to the hyper-dual angle $\tilde{\varphi}$ by using $\langle\widetilde{\mathbb{A}}, \widetilde{\mathbb{B}}\rangle_{H D}=\cos \tilde{\varphi}=\cos \varphi-\varepsilon^{*} \varphi^{*} \sin \varphi$, where $\cos \varphi=\cos \theta-\varepsilon \theta^{*} \sin \theta$ and $\sin \varphi=\sin \theta+\varepsilon \theta^{*} \cos \theta$ :

1. If

$$
\cos \varphi=0 \text { and } \varphi^{*} \neq 0,
$$

then $\theta=\frac{\pi}{2}$ and $\theta^{*}=0$. Hence, $\langle\widetilde{\mathbb{A}}, \widetilde{\mathbb{B}}\rangle_{H D}=-\varepsilon^{*} \varphi^{*}$. Thus, dual lines $\hat{d}_{1}$ and $\hat{d}_{2}$ are perpendicular but not intersecting.

2. If

$$
\varphi^{*}=0,
$$

then $\langle\widetilde{\mathbb{A}}, \widetilde{\mathbb{B}}\rangle_{H D}=\cos \varphi$. Thus, dual lines $\hat{d}_{1}$ and $\hat{d}_{2}$ are intersecting.

3. If

$$
\langle\widetilde{\mathbb{A}}, \widetilde{\mathbb{B}}\rangle_{H D}=0,
$$

then $\cos \varphi=0$ and $\varphi^{*}=0$. Hence, $\theta=\frac{\pi}{2}$ and $\theta^{*}=0$. Thus, $\hat{d}_{1}$ and $\hat{d}_{2}$ are perpendicular and intersecting lines.

4. If

$$
\langle\widetilde{\mathbb{A}}, \widetilde{\mathbb{B}}\rangle_{H D}=1,
$$

then $\theta=0$ and $\varepsilon^{*} \varepsilon \varphi^{*} \theta^{*}=0$. Thus, the following two cases can be given:

(i) If $\theta^{*}=0$, then $\varphi=0$. Hence, $\hat{d}_{1}$ and $\hat{d}_{2}$ are parallel lines.

(ii) If $\varphi^{*}=0$, then $\tilde{\varphi}=\varphi=\varepsilon \theta^{*}$.

Example 3. (Application of hyper-dual angle) Let us take the unit hyper-dual vectors $\widetilde{\mathbb{A}}=(1, \varepsilon, \varepsilon)+\varepsilon^{*}(\varepsilon, \varepsilon,-1)$ and $\widetilde{\mathbb{B}}=(0,1, \varepsilon)+\varepsilon^{*}(2 \varepsilon, \varepsilon,-1)$ that can be written in the form $\widetilde{\mathbb{A}}=\hat{A}+\varepsilon^{*} \hat{A}^{*}$ and $\widetilde{\mathbb{B}}=\hat{B}+\varepsilon^{*} \hat{B}^{*}$ for $\hat{A}=(1, \varepsilon, \varepsilon)$, $\hat{A}^{*}=(\varepsilon, \varepsilon,-1), \hat{B}=(0,1, \varepsilon)$ and $\hat{B}^{*}=(2 \varepsilon, \varepsilon,-1)$. Hyper-dual angle $\tilde{\varphi}$ between $\widetilde{\mathbb{A}}$ and $\widetilde{B}$ will be obtained as

$$
\begin{aligned}
\langle\widetilde{\mathbb{A}}, \widetilde{\mathbb{B}}\rangle_{H D} & =\langle\hat{A}, \hat{B}\rangle_{D}+\varepsilon^{*}\left(\left\langle\hat{A}, \hat{B}^{*}\right\rangle_{D}+\left\langle\hat{A}^{*}, \hat{B}\right\rangle_{D}\right) \\
& =\cos \varphi-\varepsilon^{*} \varphi^{*} \sin \varphi \\
& =\cos \left(\varphi+\varepsilon^{*} \varphi^{*}\right) \\
& =\cos \tilde{\varphi} .
\end{aligned}
$$

Here; $\langle\hat{A}, \hat{B}\rangle_{D}$ is equal to

$$
\begin{aligned}
\langle\hat{A}, \hat{B}\rangle_{D} & =\langle(1, \varepsilon, \varepsilon),(0,1, \varepsilon)\rangle_{D} \\
& =\varepsilon
\end{aligned}
$$

and from the Equation (2.15), the equality

$$
\begin{aligned}
\langle\hat{A}, \hat{B}\rangle_{D} & =\cos \varphi \\
& =\cos \left(\theta+\varepsilon \theta^{*}\right) \\
& =\cos \theta-\varepsilon \theta^{*} \sin \theta
\end{aligned}
$$


can be given. Since Equations (3.57) and (3.58) are equal, we get

$$
\theta=\frac{\pi}{2} \text { and } \theta^{*}=-1 \text { so } \varphi=\frac{\pi}{2}-\varepsilon
$$

We can obtain $\left\langle\hat{A}, \hat{B}^{*}\right\rangle_{D}+\left\langle\hat{A}^{*}, \hat{B}\right\rangle_{D}$ as

$$
\begin{aligned}
\left\langle\hat{A}, \hat{B}^{*}\right\rangle_{D}+\left\langle\hat{A}^{*}, \hat{B}\right\rangle_{D} & =\langle(1, \varepsilon, \varepsilon),(2 \varepsilon, \varepsilon,-1)\rangle_{D}+\langle(\varepsilon, \varepsilon,-1),(0,1, \varepsilon)\rangle_{D} \\
& =\varepsilon .
\end{aligned}
$$

Using the Taylor series expansion given by Equation (2.7), we get

$$
\begin{aligned}
\sin \varphi & =\sin \left(\theta+\varepsilon \theta^{*}\right) \\
& =\sin \theta+\varepsilon \theta^{*} \cos \theta .
\end{aligned}
$$

And using Equation (3.59) in Equation (3.61), we obtain

$$
\sin \varphi=1 .
$$

From the equality of the Equations (3.41) and (3.43), we can write

$$
\left\langle\hat{A}, \hat{B}^{*}\right\rangle_{D}+\left\langle\hat{A}^{*}, \hat{B}\right\rangle_{D}=-\varphi^{*} \sin \varphi .
$$

Inserting Equations (3.60) and (3.62) in Equation (3.63), we obtain

$$
\varepsilon=-\varphi^{*}
$$

Finally; from Equations (3.59) and (3.64), hyper-dual angle $\tilde{\varphi}$ is obtained as

$$
\tilde{\varphi}=\left(\frac{\pi}{2}-\varepsilon\right)-\varepsilon^{*} \varepsilon
$$

\section{Conclusions}

In this paper, the basic and kinematic concepts of hyper-dual numbers are given by using properties of dual numbers. The concept "dual line" is defined to represent the geometric interpretation of unit hyper-dual vectors in $\mathbb{D}^{3}$. Using these concepts, the geometric interpretations of E. Study mapping and hyper-dual angle are given. Furthermore; by taken $\left|\hat{A}^{*}\right|_{D}=1$ in the unit hyper-dual vectors set $\tilde{\mathbb{S}}=\left\{\widetilde{\mathbb{A}}=\hat{A}+\varepsilon^{*} \hat{A}^{*}:|\widetilde{\mathbb{A}}|_{H D}=1 ; \hat{A}, \hat{A}^{*} \in \mathbb{D}^{3}\right\}$, we have defined the subset $\tilde{\mathbb{S}}_{1}$. And it is shown that there exists a one to one correspondence between the points of the subset $\tilde{\mathbb{S}}_{1}$ and any two intersecting perpendicular directed lines in $\mathbb{R}^{3}$.

\section{References}

[1] Agrawal, O. P.: Hamilton operators and dual-number-quaternions in spatial kinematics. J. Mech. Mach. Theory. 22(6), 569-575 (1987).

[2] Clifford, W. K.: Preliminary sketch of biquaternions. Proc London Mathematical Society. 4(64), $381-395$ (1873).

[3] Cohen, A., Shoham, M.: Application of hyper-dual numbers to multi-body kinematics. J. Mech. Rob. 8, (2015). doi: 10.1115/1.4030588.

[4] Cohen, A., Shoham, M.: Application of hyper-dual numbers to rigid bodies equations of motion. J. Mech. Mach. Theory. 111, 76-84 (2017).

[5] Cohen, A., Shoham, M.: Principle of transference-An extension to hyper-dual numbers. J. Mech. Mach. Theory. 125, 101-110 (2018).

[6] Fike, J. A.: Numerically exact derivative calculations using hyper-dual numbers, 3rd Annual Student Joint Workshop in Simulation-Based Engineering and Design, (2009).

[7] Fike, J. A., Alonso, J. J.: The development of hyper-dual numbers for exact second-derivative calculations. 49th AIAA Aerodpace Sciences Meeting including the New Horizons Forum and Aerospace Exposition. 4-7 (2011).

[8] Fike, J. A., Alonso, J. J.: Automatic differentiation through the use of hyper-dual numbers for second derivatives. in: Lecture Notes in Computational Science and Engineering. 87(201), 163-173 (2011).

[9] Fike, J. A., Jongsma, S., Alonso, J. J., van der Weida, E.: Optimization with gradient and hessian information calculated using hyper-dual numbers. 29 AIAA Applied Aerodynamics Conference. (2011). 
[10] Kotelnikov, A. P.: Screw calculus and some applications to geometry and mechanics. Annal Imp. Univ. Kazan, Russia, (1895).

[11] Study, E.: Geometry der Dynamen. Leipzig. (1901).

[12] Veldkamp, G. R.: On the use of dual numbers, vectors and matrices in instantaneous, spatial kinematics. J. Mech. Mach. Theory. 11(2), 141-156 (1976).

[13] Yuca, G., Yaylı, Y.: Dual Transformation Between $S \hat{O}(3)$ and $S \hat{O}(2,1)$ and Its Geometric Applications. Proc. Natl. Acad. Sci. India, Sect. A Phys. Sci. 88, 267-273 (2018).

[14] Yuca, G., Yaylı, Y.: Dual Transformations and Instantaneous Screw Axes. International Journal of Mathematics Trends and Technology. 15 (2), (2014).

\section{Affiliations}

SElahatTin ASLAN

ADDRESS: Ankara University, Department of Mathematics, 06100, Ankara-Turkey.

E-MAIL: selahattinnaslan@gmail.com

ORCID ID: 0000-0001-5322-3265 